\title{
Expression and prognostic significance of CTBP2 in human gliomas
}

\author{
YONG WANG $^{1 *}$, SHUSHENG CHE $^{2 *}$, GANG CAI $^{1}$, YUCHAO HE $^{2}$, JIALEI CHEN $^{1}$ and WEI XU ${ }^{1}$ \\ ${ }^{1}$ Department of Neurosurgery, The Second Affiliated Hospital of Nantong University, Nantong, Jiangsu 226001; \\ ${ }^{2}$ Department of Neurosurgery, The Affiliated Hospital of Qingdao Medical College, \\ Qingdao University, Qingdao, Shandong 266003, P.R. China
}

Received April 6, 2015; Accepted July 1, 2016

DOI: $10.3892 / \mathrm{ol} .2016 .4998$

\begin{abstract}
Deregulated expression of C-terminal-binding protein 2 (CTBP2) has been observed previously in a number of tumors, such as hepatocellular carcinoma and prostatic cancer, in the colorectal cancer SW480 cell line and in the human embryonic kidney 293 cell line. In the present study, western blot analysis and immunohistochemistry were performed to investigate whether gliomas exhibit deregulated CTBP2 expression. Kaplan-Meier survival analyses were performed to evaluate the associations between CTBP2 expression, clinicopathological data and patient survival in glioma patients. The results revealed that CTBP2 expression was significantly upregulated in high grade glioma tissues compared with that in low grade glioma and normal brain tissues. Furthermore, increased CTBP2 expression in gliomas was significantly associated with a higher World Health Organization $(\mathrm{WHO})$ tumor grade $(\mathrm{P}<0.005)$ and poorer disease-specific survival $(\mathrm{P}<0.005)$. In conclusion, these results suggest that CTBP2 may act as an intrinsic regulator of progression in glioma cells and thus may serve as an important prognostic factor for the disease.
\end{abstract}

\section{Introduction}

Glioma is the most common type of primary malignant brain tumor (1-3). In the United States, the annual morbidity rate

Correspondence to: Dr Wei Xu, Department of Neurosurgery, The Second Affiliated Hospital of Nantong University, 6 Baby Lane North Road, Nantong, Jiangsu 226001, P.R. China

E-mail: xuweidocdoc@163.com

Dr Shusheng Che, Department of Neurosurgery, The Affiliated Hospital of Qingdao Medical College, Qingdao University, 16 Jiangsu Road, Qingdao, Shandong 266003, P.R. China

E-mail: shusheng6100@126.com

"Contributed equally

Abbreviations: CTBPs, C-terminal-binding proteins; KPS, Karnofsky performance status

Key words: gliomas, CTBP2, expression, clinical significance of glioma is $0.5 \%$. There are $\sim 18,000$ new glioma cases and 13,000 mortaloties annually. The prognosis of glioblastoma remains particularly poor and its 5-year survival rate is $<3 \%$, which is only second to pancreatic and lung cancer (4). Although anticancer regimens and surgery are used to treat glioma, tumor cells often migrate into brain parenchyma and thus patients succumb to the disease extremely quickly as invasive cells are able to escape radiation exposure and resection (5-7). Currently, no effective treatments for glioma have been identified as the potential mechanism of the disease and prognostic factors of glioma remain unclear (8). Therefore, the development of novel diagnostic methods to effectively manage gliomas is urgently required.

C-terminal-binding proteins (CTBPs) were originally identified based on their ability to bind the C-terminus of type 2/5 adenovirus 243R E1A proteins as cellular binding partners (9). CTBPs are involved in multiple biological processes via a number of transcription factors. In the nucleus, CTBPs act as transcriptional corepressors, while in the cytoplasm they regulate the endocytic membranes and fission of Golgi (10-12). In vertebrates, CTBP has two isoforms, CTBP1 and CTBP2, located at human chromosomes 4 and 10, respectively and multiple mechanisms have indicated that these proteins are also involved in the Wnt signaling pathways and cell cycle regulation $(13,14)$ by acting as direct corepressors of transcription factor (TCF)-3 and TCF-4 (15). These functions have been demonstrated in a number of human cells, including colorectal cancer cell lines (15). Although CTBP1 and CTBP2 share similarities in gene expression and function, there remains a difference between the two. A recent study revealed that dorsal root ganglia express CTBP1, whereas migrating neural crest cells express CTBP2 (16). Notably, the majority of CTBP1 mutant mice are fertile and viable, however, CTBP2 mutations lead to embryonic lethality and delayed neural development (17). CTBP2 proteins also exhibit a critical function in tumorigenesis (18). The overexpression of CTBP2 reduces phosphatase and tensin homolog levels in tumor cells (19).

At present, literature regarding the expression of CTBP2 proteins in cancer is limited; CTBP2 expression in brain tumors has not been reported previously. In the present study, CTBP2 protein expression in gliomas was investigated. This study evaluated whether CTBP2 is expressed in glioma and whether it is involved in the proliferation and differentiation of 
the disease, as well as its association with glioma grade. The aims of the present study were to investigate the association between CTBP2 expression and clinicopathological features in glioma patients and to identify potential therapeutic strategies for the disease.

\section{Patients and methods}

Patients. A total of 60 glioma patients that underwent subtotal resection at The Second Affiliated Hospital of Nantong University (Nantong, China) between February 2005 and February 2011 were included in the present study. The patient cohort included 31 male and 29 female patients with a median age of 42 years (range, 18-70 years). No patients had received preoperative chemotherapy and after the surgical incision had healed at post-operative day 15-20, all patients were treated with tridimensional radiotherapy with 2 Gy/day, 5 days/week, continuous radiotherapy for 5-7 weeks and a total dose of 60-66 Gy. A week after radiation treatment, the patients were administered oral chemotherapy consisting of lomustine at a dose of $0.005-0.006 \mathrm{mg} / \mathrm{m}^{2} /$ day, 5 days/week, with a treatment interval of 23 days and a treatment cycle of 28 days. The treatment lasted for 3-5 cycles according to the patients' tolerance. Patients that succumbed due to other diseases or causes were excluded from the study. The functional impairment of all patients was assessed using Karnofsky performance status (KPS) scores (20): 30 patients exhibited a KPS score of $<80$ and 30 patients exhibited a KPS score of $>80$. Histological grading and classification were conducted in all gliomas according to the World Health Organization (WHO) 2007 central nervous system tumor classification system (21). A total of 25 patients exhibited low grade (I-II) malignant glioma and 35 patients exhibited high grade (III-IV) malignant glioma. A total of 8 brain tissues that were obtained from patients undergoing intracranial decompression due to brain trauma served as the control group. Postoperative pathology confirmed that all control tissues exhibited no glial cell proliferation.

Tissue preparation. A total of 60 glioma tissue samples were obtained from glioma patients that underwent subtotal resection and 8 control brain tissues were obtained from brain injury patients that underwent intracranial decompression. Necrotic tissue was removed from specimens immediately following isolation. Each patient specimen was divided into two sections: One section was immediately frozen at $-80^{\circ} \mathrm{C}$ for subsequent use and the other section was fixed with $10 \%$ neutral formalin, paraffin-embedded and cut into 5-mm sections. Consent for the collection of specimens was obtained from all patients and the study was approved by the Ethics Committee of The Second Affiliated Hospital of Nantong University.

Reagents. Mouse anti-human monoclonal CTBP2 antibody (catalog no. ab128871) was obtained from Abcam (Cambridge, MA, USA), biotin-labeled anti-mouse immunoglobulin G (IgG; catalog no. BA1001) was obtained from Boster Biological Engineering Co., Ltd., (Wuhan, China). $\beta$-actin monoclonal antibody (catalog no. AF0003) was purchased from Beyotime Institute of Biotechnology (Shanghai, China). Polymerized peroxidase-labeled goat anti-mouse IgG (catalog no. A0216; Beyotime Institute of Biotechnology) was used for immunohistochemical staining. Donkey anti-mouse IgG biotinylated antibody (catalog no. BAF018; R\&D Systems, Shanghai, China) and western immunoblot kit (sodium dodecyl sulfate-polyacrylamide gel electrophoresis gel preparation kit; catalog no. P0012A; Beyotime Institute of Biotechnology) and RIPA lysis solution (catalog no. P0013C; Beyotime Institute of Biotechnology) were used for western blotting.

Western blot analysis. Total protein was extracted from the frozen tumor specimens using a Nuclear and Cytoplasmic Protein Extraction kit and stored at $-70^{\circ} \mathrm{C}$ after the concentration was determined by ultraviolet spectrophotometry. Separating gel and stacking gel, as well as the electrophoresis buffer, were configured according to the instructions of the western immunoblot kit (22), followed by sample loading and electrophoresis. After electrophoresis, proteins were transferred to nitrocellulose membranes, blocked with skimmed milk powder and incubated with mouse anti-human monoclonal CTBP2 antibody (dilution, $1: 1,000$ ) at $4^{\circ} \mathrm{C}$ overnight. The membrane was washed 3 times, for $10 \mathrm{~min}$ each, followed by incubation with HRP-labeled donkey anti-mouse IgG at $4^{\circ} \mathrm{C}$ for $\sim 4 \mathrm{~h}$. Next, the membrane was washed and DAB (Beyotime Institute of Biotechnology) was added as the chromogen. After washing the membrane, enhanced chemiluminescence reagent (Beyotime Institute of Biotechnology) was added for development. $\beta$-actin served as an internal control (dilution, 1:1,000). All the western band densities were analyzed using ImageQuant LAS 4000 (GE Healthcare Life Science, Shanghai,China) and Quantity One v4.62 software (Bio-Rad, Hercules, CA, USA).

Immunohistochemical staining. Formalin-fixed paraffin embedded 5-mm sections were dewaxed conventionally and treated with $3 \% \mathrm{H}_{2} \mathrm{O}_{2}$ to inactivate the endogenous peroxidases, followed by blocking with fetal bovine serum (Gibco; Thermo Fisher Scientific, Waltham, MA, USA). Following incubation with mouse anti-human monoclonal CTBP2 antibody (dilution, 1:100) overnight at room temperature the sections were incubated with polymerized peroxidase-labeled goat anti-mouse IgG (dilution, 1:100). DAB was then added as the chromogen and sections were dehydrated for transparent mounting. For the evaluation of CTBP2 expression in different grades of tumors, 10 randomly selected visual fields per section were examined at x400 magnifications on a light microscope (Leica, Wetzlar, Germany).

Cells that exhibited yellow, brown or reddish brown cytoplasmic staining were regarded as positively-stained cells. Immunohistochemistry scores were calculated based on the staining intensity and the percentage of positive cells. Staining intensity was defined as follows: 0, negative staining; 1, weak staining; 2, moderate staining; and 3, strong staining. The number of positive cells was defined as follows: $0,0 \%$ positive cells; $1,<25 \%$ positive cells; $2,25-50 \%$ positive cells; $3,51-80 \%$ positive cells; and $4,>80 \%$ positive cells. Five fields of vision were randomly selected under high magnification (x400) and 200 cells were counted in each field of vision. The number of positive cells were counted to calculate the mean. Based on the sum of the staining intensity and number of positive cell scores, immunohistochemistry staining scores 
Table I. CTBP2 expression scores in NB tissue and WHO grade I-II and III-IV gliomas.

\begin{tabular}{lrrrrr}
\hline & & \multicolumn{4}{c}{ CTBP2 expression score, $\mathrm{n}$} \\
\cline { 3 - 6 } Group & $\mathrm{n}$ & & + & ++ & +++ \\
\hline NB & 8 & 5 & 2 & 1 & 0 \\
Grade I-II & 25 & 2 & 6 & 14 & 3 \\
Grade III-IV & 35 & 0 & 4 & 11 & 20 \\
\hline
\end{tabular}

Positive CTBP2 expression rate was positively correlated with WHO glioma grade $(\mathrm{P}=0.003)$. CTBP2, C-terminal-binding protein 2; NB, normal brain; WHO, World Health Organization.

Table II. Association between CTBP2 expression and clinicopathological characteristics of 60 glioma patients.

\begin{tabular}{|c|c|c|c|c|}
\hline \multirow{2}{*}{$\begin{array}{l}\text { Clinicopathological } \\
\text { parameter }\end{array}$} & \multirow[b]{2}{*}{$\mathrm{n}$} & \multicolumn{2}{|c|}{ CTBP2 expression } & \multirow[b]{2}{*}{ P-value } \\
\hline & & Low, n & High, $\mathrm{n}$ & \\
\hline Gender & & & & 0.438 \\
\hline Male & 31 & 14 & 17 & \\
\hline Female & 29 & 16 & 13 & \\
\hline Age, years & & & & 0.436 \\
\hline$<50$ & 33 & 18 & 15 & \\
\hline$>50$ & 27 & 12 & 15 & \\
\hline KPS score & & & & 0.302 \\
\hline$<80$ & 30 & 13 & 17 & \\
\hline$>80$ & 30 & 17 & 13 & \\
\hline Tumor location & & & & 0.501 \\
\hline Frontal & 27 & 12 & 15 & \\
\hline Temporal & 23 & 11 & 12 & \\
\hline Other & 10 & 7 & 3 & \\
\hline Tumor diameter, cm & & & & 0.602 \\
\hline$<4$ & 26 & 12 & 14 & \\
\hline$>4$ & 34 & 18 & 16 & \\
\hline WHO grade & & & & 0.001 \\
\hline I-II & 25 & 19 & 6 & \\
\hline III-IV & 35 & 11 & 24 & \\
\hline
\end{tabular}

CTBP2, C-terminal-binding protein 2; WHO, World Health Organization; KPS, Karnofsky performance status.

were as follows: $0-2$, negative $(-) ; 3$, weak $(+)$; 4-5, moderate $(++)$; and 6-7, strong $(+++)$.

Statistical analysis. All data analysis was performed using SPSS 19.0 statistical software (SPSS, Inc., Chicago, IL, USA) and $\mathrm{P}<0.05$ was considered to indicate a statistically significant difference. Data are expressed as the mean \pm standard deviation. The correlation between CTBP 2 protein expression and clinicopathological parameters was analyzed using the $\chi^{2}$ test and Spearman's rank correlation. One way analysis of
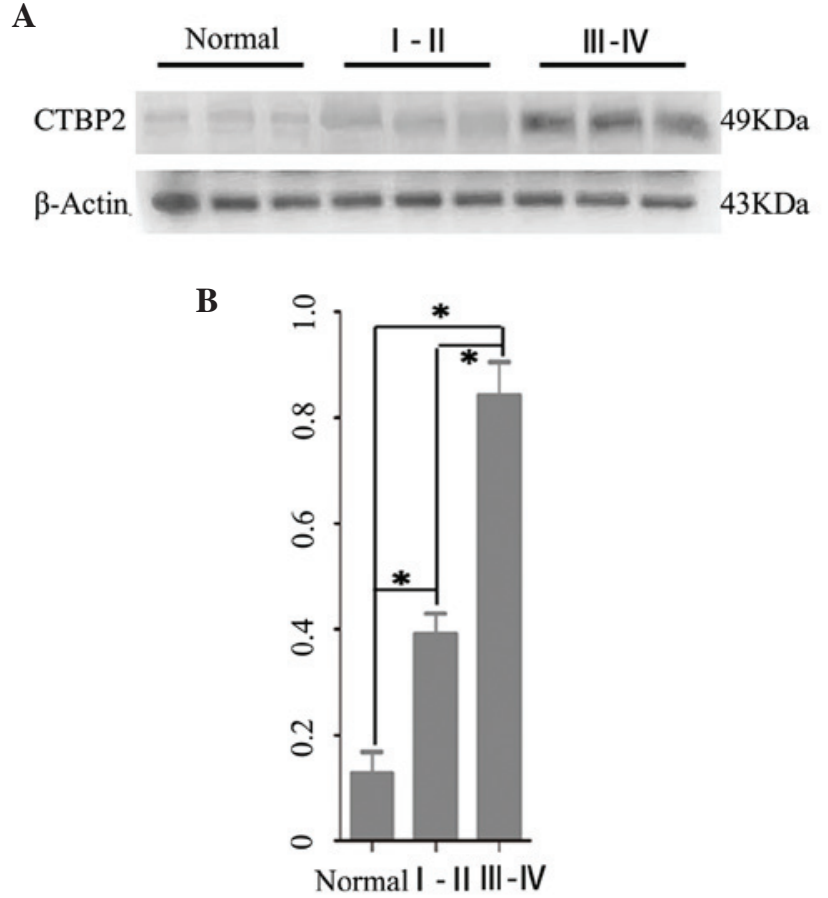

Expression of CTBP2 in normal brain and different grades gliomas

Figure 1. CTBP2 protein expression in normal brain tissues and different grades of glioma was analyzed by western blot. (A) Western blot demonstrating that CTBP2 protein expression was higher in glioma tissues (World Health Organization grades I-II and III-IV) than normal brain tissues. (B) Western blot quantification showing CTBP2 protein expression levels in glioma and normal brain tissues. ${ }^{*} \mathrm{P}<0.05$. CTBP2, C-terminal-binding protein 2 .

variance was used to compare differences between groups. Kaplan-Meier survival curves were generated and survival rates were assessed using the log-rank test.

\section{Results}

CTBP2 protein expression is higher in glioma tissues than normal brain tissues. Western blot analysis revealed that CTBP2 protein expression levels in both the high grade (WHO III-IV) glioma (0.843 \pm 0.354$)$ and low grade (WHO I-II) glioma groups $(0.402 \pm 0.132)$ were significantly higher than that in brain tissues of the normal brain tissue control group $(0.134 \pm 0.073)(\mathrm{P}=0.005 ; \mathrm{P}=0.008)$. Furthermore, the CTBP2 protein expression level in the high grade glioma group was significantly higher than that in the low grade glioma group $(\mathrm{P}=0.042)$ (Fig. 1).

CTBP2 positive expression rate is higher in glioma tissues than normal brain tissues. Immunohistochemical staining revealed that CTBP2 protein was predominantly localized in the nucleus of glioma cells and was also marginally expressed in the cytoplasm (Fig. 2). The positive expression rate of CTBP2 in all glioma patients was $96.67 \%$, while the positive expression rate of CTBP2 in the control group was $37.5 \%$. The strong positive expression rate of CTBP 2 in the control, low grade glioma and high grade glioma groups was $0.00,12.00$ and $57.14 \%$, respectively. The negative expression rate of CTBP2 was $62.5 \%$ in the control group and $0.00 \%$ in 
A
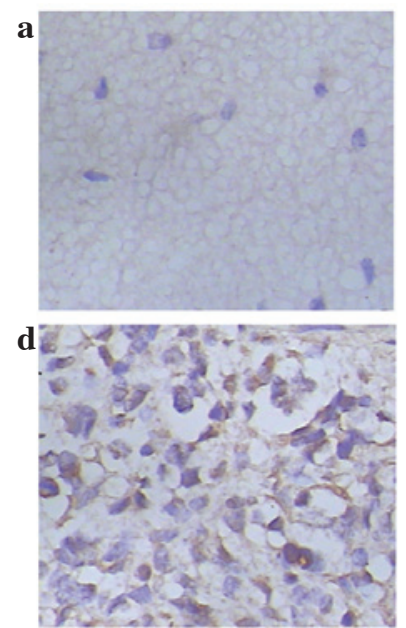
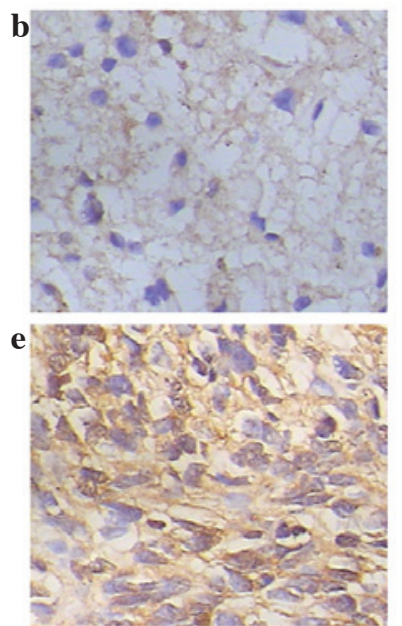

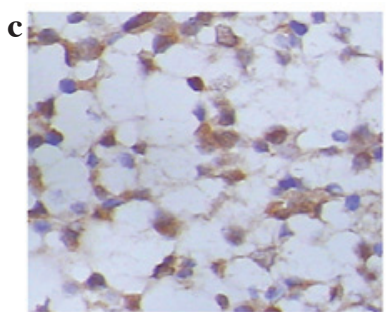

B

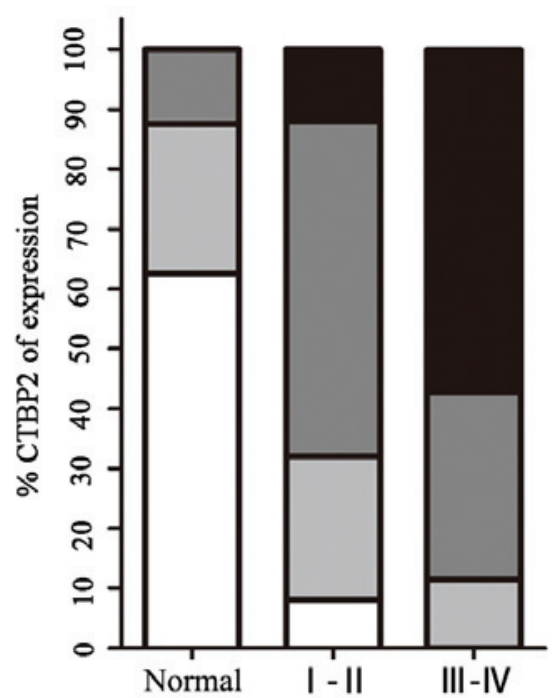

\section{Expression of CTBP2 in normal brain and different grades}

Figure 2. Immunohistochemical staining for CTBP2 in representative samples of (Aa) normal brain tissue, (Ab) WHO grade I glioma, (Ac) WHO grade II glioma, (Ad) WHO grade III glioma and (Ae) WHO grade IV glioma (magnification, x400) revealing that high-grade glioma exhibits stronger CTBP2 expression than lower grade gliomas and normal brain tissue. (B) Overall CTBP2 expression levels were significantly higher in WHO III-IV glioma tissues compared with WHO I-II glioma tissues and normal brain tissues $(\mathrm{P}<0.05)$. CTBP2, C-terminal-binding protein 2; WHO, World Health Organization.

\section{Survival Function}

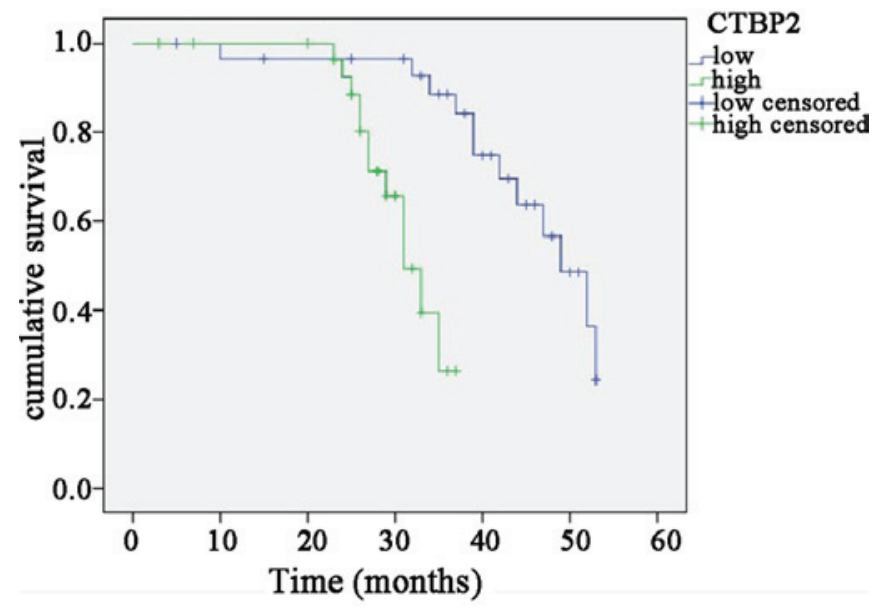

Figure 3. Kaplan-Meier survival curves for glioma patients with high or low expression of CTBP2. CTBP2, C-terminal-binding protein 2. the high grade glioma group $(\mathrm{P}<0.05)$. These results demonstrated that the positive expression of CTBP2 in the high grade (WHO III-IV) glioma group was significantly higher than that in the low grade (WHO I-II) glioma and control groups $(\mathrm{P}=0.003)$ (Table I).

High CTBP2 expression is associated with high glioma grade and poor prognosis of glioma patients. All glioma patients were divided into high CTBP2 expression (number of positive cells, $>50 \%$ ) and low CTBP2 expression (number of positive cells, $<50 \%$ ) groups according to the expression of CTBP2 identified by immunohistochemistry. No significant associations between CTBP2 expression and gender, age, tumor size, location or KPS scores were identified. However, CTBP2 expression was significantly correlated with pathological WHO glioma grade $(\mathrm{P}=0.001)$ (Table II). Notably, survival analysis revealed that the low CTBP2 expression group exhibited a significantly longer survival time and better prognosis than the high CTBP2 expression group ( $\mathrm{P}=0.001)$ (Fig. 3). 


\section{Discussion}

Despite recent advances in diagnostic modalities and therapeutic strategies, such as radiation therapy and chemotherapeutic regimens, glioma remains one of the most lethal types of human cancer $(23,24)$. The median survival time of glioma patients is $<2$ years and the 5-year survival rate is among the lowest for all cancers, at $<3 \%$ (25). In recent years, studies have been conducted to identify a potential therapeutic target for human malignant gliomas. An increased understanding of the biology and molecular mechanisms underlying glioma development and progression is required for advances in the treatment of glioma.

CTBPs function in the nucleus as transcriptional co-repressors via their interaction with adenovirus E1A (26). Extensive molecular and cellular analyses have identified CTBPs as regulators of pathways that are critical for tumor initiation, progression, cell migration, cell apoptosis and response to therapy $(15,27)$. However, data regarding the expression and regulation of CTBPs in human cancers and the involvement of CTBPs in glioma is limited. By contrast to CTBP1, which is expressed from embryo to adult, CTBP2 is predominantly expressed during embryogenesis (16). Additionally, previous studies have demonstrated that the CTBP2 protein is involved in pathways associated with tumorigenesis, including transforming growth factor- $\beta$ and Wnt signaling pathways and cell cycle regulation $(10,11)$. Thus, these findings are consistent with those of the present study which revealed that CTBP2 is highly expressed in malignant gliomas and increases with ascending tumor WHO grade. However, CTBP2 expression in astrocytic tumors has not yet been fully characterized and to date its function in glioma cells has not been reported.

The results of the present study revealed that CTBP2 expression was markedly increased in malignant gliomas and directly correlated with WHO glioma grade. Immunostaining revealed that CTBP2 was predominantly localized in the nuclei. By evaluating the association between CTBP2 expression and clinicopathological variables, it was demonstrated that CTBP2 expression correlated with WHO glioma grade and patient survival. The increased CTBP 2 expression in gliomas was significantly associated with higher WHO grade and poorer disease-specific survival of patients $(\mathrm{P}=0.001)$. However, further studies that investigate CTBP2 expression and its potential prognostic value are required in the future.

Although CTBP2 has been reported to be highly expressed in a number of cancers $(10,28)$, its expression in glioma tissues with varying degrees of malignancy has not been examined. A previous study demonstrated that CTBP2, as a coregulator of nuclear transcription, directly interacts with various targets to promote astrocytical activation and proliferation (29). We postulate that CTBP2 may be re-expressed in glioma, and that the pattern of expression may correlate with the degree of malignant potential. This hypothesis was confirmed by the current study, which revealed that increased CTBP2 expression correlates with a higher WHO glioma grade and poor patient survival. However, the detailed mechanism underlying transcriptional regulation of CTBP2 in glioma remains unclear. CTBP2 functions as a corepressor for a substantial number of transcriptional repressors and consequently regulates diverse cellular processes. p53, which is a target of CTBP2, suppresses the sensitivity of breast cancer cells to mechanistically diverse cancer chemotherapeutic agents, (30). Furthermore, CTBP2 is involved in the repression of p53-inducible pro-apoptotic genes, including Bax and Noxa, which are involved in the induction of cellular apoptosis $(31,32)$. These findings support the results that CTBP2 was highly expressed in glioblastomas compared with low grade gliomas and normal brain tissues. Recent studies have demonstrated that CTBP2, as a negative transcriptional regulator of $\mathrm{p} 16^{\mathrm{INK} 4 \mathrm{~A}}$, may modulate cell proliferation and contribute to the progression of esophageal squamous cell carcinoma (33). These findings indicate that CTBP2 provides prognostic information and thus, targeting CTBP2 may present a novel approach for glioma treatment. However, the biological functions of CTBP2 and its involvement in tumorigenesis require further investigation. In addition, the mechanism by which CTBP2 and p53 or p16 coordinately modulate the growth of glioma also requires investigation in the future.

In conclusion, the results of the present study revealed that the expression of CTBP 2 was increased in high grade glioblastomas compared with low grade glioblastomas and normal brain tissue and that high CTBP2 expression was associated with a poorer disease-specific survival. Gliomas exhibit poor prognosis due to their invasive potential and resistance to current therapeutic modalities. The results of this study indicate that CTBP2 may present a potential diagnostic marker and a novel therapeutic target in the treatment of glioma.

\section{Acknowledgements}

The authors would like to thank the Department of Neurosurgery, Affiliated Hospital of Medical College Qingdao University (Qingdao, China) for providing technical assistance and equipment support.

\section{References}

1. Claes A, Idema AJ and Wesseling P: Diffuse glioma growth: A guerilla war. Acta Neuropathol 114: 443-458, 2007.

2. Wen PY, Fine HA, Black PM, Shrieve DC, Alexander E III and Loeffler JS: High-grade astrocytomas. Neurol Clin 13: 875-900, 1995.

3. Kleihues P, Soylemezoglu F, Schäuble B, Scheithauer BW and Burger PC: Histopathology, classification, and grading of gliomas. Glia 15: 211-221, 1995.

4. Ostrom QT, Gittleman H, Farah P, et al: CBTRUS statistical report: Primary brain and central nervous system tumors diagnosed in the United States in 2006-2010. Neuro Oncol 15 (Suppl 2): ii1-ii56, 2013.

5. Bao S, Wu Q, Li Z, Sathornsumetee S, Wang H, McLendon RE, Hjelmeland AB and Rich JN: Targeting cancer stem cells through L1CAM suppresses glioma growth. Cancer Res 68: 6043-6048, 2008.

6. Yuan X, Curtin J, Xiong Y, Liu G, Waschsmann-Hogiu S, Farkas DL, Black KL and Yu JS: Isolation of cancer stem cells from adult glioblastoma multiforme. Oncogene 23: 9392-9400, 2004.

7. Wen PY and Kesari S: Malignant gliomas in adults. N Engl J Med 359: 492-507, 2008.

8. Holland EC: Glioblastoma multiforme: The terminator. Proc Natl Acad Sci USA 97: 6242-6244, 2000.

9. Boyd JM, Subramanian T, Schaeper U, La Regina M, Bayley S and Chinnadurai $\mathrm{G}$ : A region in the $\mathrm{C}$-terminus of adenovirus $2 / 5$ E1a protein is required for association with a cellular phosphoprotein and important for the negative modulation of T24-ras mediated transformation, tumorigenesis and metastasis. EMBO J 12: 469-478, 1993.

10. Bergman LM and Blaydes JP: C-terminal binding proteins: Emerging roles in cell survival and tumorigenesis. Apoptosis 11: 879-888, 2006 
11. Chinnadurai G: The transcriptional corepressor CtBP: A foe of multiple tumor suppressors. Cancer Res 69: 731-734, 2009.

12. Corda D, Colanzi A and Luini A: The multiple activities of CtBP/BARS proteins: The Golgi view. Trends Cell Biol 16: 167-173, 2006.

13. Meloni AR, Smith EJ and Nevins JR: A mechanism for $\mathrm{Rb} / \mathrm{p} 130$-mediated transcription repression involving recruitment of the CtBP corepressor. Proc Natl Acad Sci USA 96: 9574-9579, 1999.

14. Izutsu K, Kurokawa M, Imai Y, Maki K, Mitani K and Hirai H: The corepressor CtBP interacts with Evi-1 to repress transforming growth factor beta signaling. Blood 97: 2815-2822, 2001.

15. Hamada F and Bienz M: The APC tumor suppressor binds to $\mathrm{C}$-terminal binding protein to divert nuclear beta-catenin from TCF. Dev Cell 7: 677-685, 2004.

16. Van Hateren N, Shenton T and Borycki AG: Expression of avian C-terminal binding proteins (Ctbp1 and Ctbp2) during embryonic development. Dev Dyn 235: 490-495, 2006.

17. Hildebrand JD and Soriano P: Overlapping and unique roles for $\mathrm{C}$-terminal binding protein $1(\mathrm{CtBP} 1)$ and $\mathrm{CtBP} 2$ during mouse development. Mol Cell Biol 22: 5296-5307, 2002.

18. Chinnadurai G: $\mathrm{CtBP}$, an unconventional transcriptional corepressor in development and oncogenesis. Mol Cell 9: 213-224, 2002

19. Paliwal S, Kovi RC, Nath B, Chen YW, Lewis BC and Grossman SR: The alternative reading frame tumor suppressor antagonizes hypoxia-induced cancer cell migration via interaction with the $\mathrm{COOH}$-terminal binding protein corepressor. Cancer Res 67: 9322-9329, 2007.

20. Mor V, Laliberte L, Morris JN and Wiemann M: The Karnofsky Performance Status Scale. An examination of its reliability and validity in a research setting. Cancer 53: 2002-2007, 1984.

21. Fuller GN and Scheithauer BW: The 2007 Revised World Health Organization (WHO) Classification of Tumours of the Central Nervous System: Newly codified entities. Brain Pathol 17: 304-307, 2007

22. Liu R, Liu X, Zheng Y, Gu J, Xiong S, Jiang P, Jiang X, Huang E, Yang Y, Ge D and Chu Y: MicroRNA-7 sensitizes non-small cel lung cancer cells to paclitaxel. Oncol Lett 8: 2193-2200, 2014.

23. Soffietti R, Bertero L, Pinessi L and Rudà R: Pharmacologic therapies for malignant glioma: A guide for clinicians. CNS Drugs 28: 1127-1137, 2014.
24. Wu CX, Lin GS, Lin ZX, Zhang JD, Chen L, Liu SY, Tang WL, Qiu XX and Zhou CF: Peritumoral edema on magnetic resonance imaging predicts a poor clinical outcome in malignant glioma. Oncology letters 10: 2769-2776, 2015.

25. Chen J, Li Y, Yu TS, McKay RM, Burns DK, Kernie SG and Parada LF: A restricted cell population propagates glioblastoma growth after chemotherapy. Nature 488: 522-526, 2012.

26. Kovi RC, Paliwal S, Pande S and Grossman SR: An ARF/CtBP2 complex regulates $\mathrm{BH} 3$-only gene expression and p53-independent apoptosis. Cell Death Differ 17: 513-521, 2010.

27. Mirnezami AH, Campbell SJ, Darley M, Primrose JN, Johnson PW and Blaydes JP: Hdm2 recruits a hypoxia-sensitive corepressor to negatively regulate p53-dependent transcription. Curr Biol 13: 1234-1239, 2003

28. Chan CB, Liu X, Jang SW, Hsu SI, Williams I, Kang S, Chen J and Ye K: NGF inhibits human leukemia proliferation by downregulating cyclin A1 expression through promoting acinus/CtBP2 association. Oncogene 28: 3825-3836, 2009.

29. Hübler D, Rankovic M, Richter K, Lazarevic V, Altrock WD Fischer KD, Gundelfinger ED and Fejtova A: Differential spatial expression and subcellular localization of CtBP family members in rodent brain. PLoS One 7: e39710, 2012.

30. Birts CN, Harding R, Soosaipillai G, Halder T, Azim-Araghi A, Darley M, Cutress RI, Bateman AC and Blaydes JP: Expression of CtBP family protein isoforms in breast cancer and their role in chemoresistance. Biol Cell 103: 1-19, 2010.

31. Paliwal S, Pande S, Kovi RC, Sharpless NE, Bardeesy N and Grossman SR: Targeting of C-terminal binding protein (CtBP) by ARF results in p53-independent apoptosis. Mol Cell Biol 26: 2360-2372, 2006.

32. Muniz VP, Barnes JM, Paliwal S, Zhang X, Tang X, Chen S, Zamba KD, Cullen JJ, Meyerholz DK, Meyers S, et al: The ARF tumor suppressor inhibits tumor cell colonization independent of p53 in a novel mouse model of pancreatic ductal adenocarcinoma metastasis. Mol Cancer Res 9: 867-877, 2011.

33. Guan C, Shi H, Wang H, Zhang J, Ni W, Chen B, Hou S, Yang X, Shen A and Ni R: CtBP2 contributes to malignant development of human esophageal squamous cell carcinoma by regulation of p16INK4A. J Cell Biochem 114: 1343-1354, 2013. 